\title{
Oral Malignant melanoma in an HIV patient
}

Chrispinus Mumena

School of Dentistry, College of Medicine and Health Sciences, University of Rwanda

Correspondence email: mumenac@gmail.com

\begin{abstract}
Oral malignant melanomas are aggressive tumors in head and neck region, rarely seen in HIV patients. By suppressing immunity, HIV might be increasing the severity of malignant tumors. Oral malignant melanomas are associated with poor prognosis, due to their aggressiveness. Of clinical importance, the prognosis of oral malignant melanomas in HIV patient is still not well understood. Presented is a 44-year-old female, HIV seropositive with a swelling on the lower lip for five months. Management involved antiretroviral therapy, surgical excision of the lesion and regional lymph nodes, with chemotherapy. Avoidance of preoperative insult and aggressive therapy offers a relatively better prognosis. Irrespective of lesion size, aggressive treatment is needed for oral malignant melanoma.
\end{abstract}

Keywords: Mouth Diseases, Melanoma, Histopathology, Non-communicable Diseases, Epidemiology, AIDS and Head and Neck

\section{Introduction}

Human Immunodeficiency Virus (HIV) infection is associated with the development of malignant lesions including melanomas.[1] HIV alters the progression of malignant tumors.[2] Oral malignant melanomas are rare aggressive neoplasms of the head and neck region,[3] usually associated with poor prognosis. The five-year survival rate is below $50 \%$, and it depends on stage and site of the lesions.[4]However, the prognosis of oral melanomas superimposed with HIV infection is not yet clear.

Globally, oral malignant melanomas have an incidence rate of $0.2 \%$ [5] to $8 \%[4]$ and account for $1.6 \%$ of all head and neck cancers,[5] $2 \%$ of all mucocutaneous melanomas[6] and $0.5 \%$ of all oral malignancies.[6] About $80 \%$ of all oral melanomas occur on the mucosa of the upper jaw.[3,7] Palate and maxillary gingiva are the most commonly affected sites followed by mandibular gingiva, buccal mucosa, the floor of mouth and tongue. [8] Most affected age is between 40 and 70 years, [8] occurring more in males than female.[9] According to clinical presentation, melanomas can clinically be grouped into three stages. Stage 1, the lesion is localized in the primary site, stage 2 the lesion involves lymph nodes, and stage 3, the lesion has hematogenous spread. [4] An HIV seropositive patient with oral malignant melanoma confined on the lip is presented.

\section{Case Presentation}

A 44-year-old lady reported with five months' swelling on the lower lip. Origin of the swelling was a small nodule that she frequently scratched, and slowly increased in size in tandem with time. Two months later she sought treatment from the Hospital but disappeared before the biopsy was done. She eventually resumed treatment four months later. Her husband was deceased four years ago with an unconfirmed diagnosis of HIV. The woman appeared with malaise, weak, and pale. Extra-oral examination revealed a firm black pedunculated swelling on the vermillion border of the lower lip that extended to skin over the lower lip. The swelling measured $5 \mathrm{x}$ $4 \mathrm{~cm}$ in the greatest dimension, ulcerated, non-tender, and bled spontaneously. The skin around the swelling was black in colour, with covered width of $2 \mathrm{~cm}$ from the tumor margin. There was a solitary palpable left submandibular lymph node that was mobile, nontender, and measured $3 \mathrm{~cm}$ in greatest dimension. Intraoral examination revealed mixed oral lesions of hairy and atrophic candidiasis on the dorsum of the tongue. The rest of oral mucosa appeared normal. Excision biopsy was planned, and the patient transferred for HIV serological testing. Antiretroviral drugs were initiated immediately after positive HIV test. Patient's general health improved drastically following the use of antiretroviral drugs. This improvement allowed progress of planned excision biopsy. Lesion on the lip and submandibular lymph node were widely excised. A surgical margin of $1 \mathrm{~cm}$ from black pigmentation around the swelling was considered. Both specimens were delivered for histopathology. The patient was followed up to monitor the progress. The histopathological results of tumor on the lip revealed malignant melanoma and tumor-free margins. The lymph node was almost entirely invaded by a characteristic melanoma composed of round, oval to spindle cells, very atypical and 
produced large amounts of melanin. The lymph node capsule was apparently not invaded. The patient was sent for adjuvant chemotherapy, tracked for four years and afterwards lost to tracking.

\section{Discussion}

Co-existence of HIV and malignant melanoma is increasing. The occurrence of large malignant melanoma on the lip is rarely seen. This is the uniqueness of this case report. Oral melanomas are usually diagnosed at an advanced stage. Often at this stage, they present as an ulcerated, painful, and bleeding swellings. $[5,8,9]$ The reported patient in our case, presented with a tumour $5 \times 4$ $\mathrm{cm}$ involving submandibular lymph node. Management of aggressive tumours that are diagnosed at advanced stage present significant challenges, requiring more expertise, and resources, and the prognosis of the patient may not be favourable.

Late diagnosis may be the outcome of delayed reporting for treatment. In this, patient delayed notification and withdrawal from treatment were noted. However, fear of the associated HIV status was the primary cause of delayed reporting. This justifies that apparent danger of death and /or extreme discomfort may be possible reasons to seek treatment. Lesion on the vermilion border and its size is a cosmetic challenge. In this case, a patient covered herself with traditional African Dressing famous by the name of "Khanga".

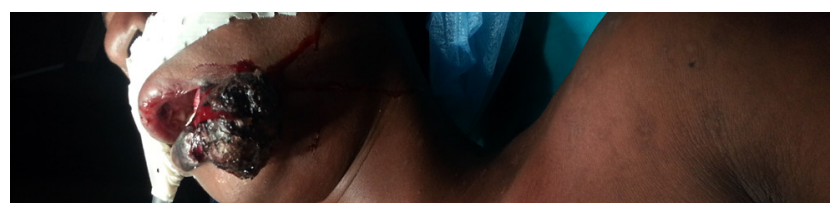

Figure 1. Picture of the patient before surgery

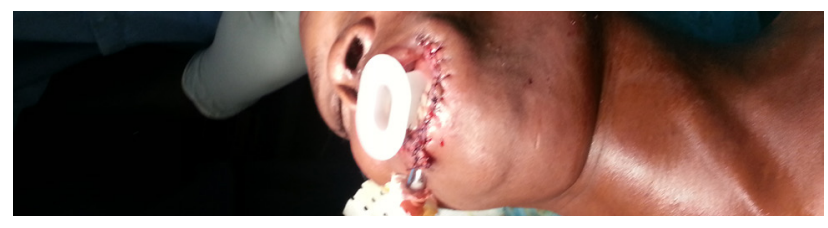

Figure 2. Picture of the patient after surgery

Oral cavity presents anatomical limitations, which is a challenge to achieve surgical clearance of advanced tumor.[8] In this patient, tumor and the palpable solitary submandibular lymph node were widely excised with a surgical margin of $1 \mathrm{~cm}$ from black depigmentation. Chemotherapy was considered after surgery. Treatment modalities of primary and metastatic melanoma are surgery, radiotherapy, chemotherapy, and immunotherapy or a combination of them.[8-10] Melanomas are relatively radioresistant. Radiotherapy[10,11] and chemotherapy[4,8,11] are not the primary treatment option. Surgery performed alone [4] or followed by radiation and /or chemotherapy $[4,8,11]$ provides a probability of cure in primary lesions, and in cases of lymph node involvement. Surgery must be done with more than 1-inch $(2-5 \mathrm{~cm})$ margin of clinical healthy skin or mucosa for favorable results. Radiotherapy is given after surgery, yield good results. $[4,10]$ In compromised patients such as elderly or those with HIV, chemotherapy is recommended before surgery to enable tumor shrinkage.[8] In the presence of metastases, chemotherapy is the cornerstone of treatment,[11] making it preferred treatment for stage IV melanomas. $[8,11]$

Malignant melanomas show good prognosis if diagnosed in early stage.[12] Metastasis is seen in late stages and occurs through hematogenous, lymphatic, perineural and trans-ductal routes.[13] In the advanced stage, median survival is two years, while the 5-year prognosis is sometimes less than 20\%.[14] Some patients are reported to die within few months after initiation of treatment.[12] Mystically, the patient survived four years without complications. Probably avoidance of incisional biopsy, wide excision of the tumor, and the palpable solitary submandibular lymph node, and postoperative chemotherapy may be the reasons behind such relatively better patient survival.

\section{Conclusion}

Avoidance of preoperative incisional biopsy is crucial for the benefit of a melanoma patient. The site of the lesion may contribute to the prognosis of the patient. Lesion on the lip may not be as aggressive as those on the gums or palate. All melanoma must receive aggressive treatment. Achieving total tumor clearance by surgery must be the objective of treatment irrespective of the size, and site of the lesion and immunological status of the patient.

Acknowledgments We would like to acknowledge Rwanda Military Hospital, where the surgery was done, King Faisal Hospital for histopathologic processing and College of Medicine and Health Sciences of the University of Rwanda, where the author works.

Conflict of interests This article has no any conflict of interest; it is shared to air our knowledge so that more information on lip melanomas is made available to all practitioners.

\section{References}

1. Butt FMA, Chindia ML, Rana F, Machigo FG. Pattern of head and neck malignant neoplasms in HIV-infected patients in Kenya. Int J Oral Maxillofac Surg. 2008;37:907-11. 
2. Rodrigues LKE, Klencke BJ, Vin-Christian K, Berger TG, Crawford RI, Miller JR, et al. Altered clinical course of malignant melanoma in HIVpositive patients. Arch Dermatol. 2002;138:765-70.

3. Manigandan T, Sagar Gv, Amudhan A, Hemalatha $\mathrm{V}$, Babu Na. Oral malignant melanoma: A case report with review of literature. Contemp Clin Dent. 2014;5:415.

4. Mendenhall WM, Amdur RJ, Hinerman RW, Werning JW, Villaret DB, Mendenhall NP. Head and Neck Mucosal Melanoma. 2005;28:626-30.

5. Ahmadi-motamayel F, Falsafi P. Report of a rare and aggressive case of oral malignant melanoma. 2013;47-51.

6. Feller L, Khammissa RAG, Lemmer J. A Review of the Aetiopathogenesis and Clinical and Histopathological Features of Oral Mucosal Melanoma. Sci World J. 2017;2017:1-7.
7. Ebenezer J. Malignant melanoma of the oral cavity Ebenezer J - Indian J ... Related articles Malignant melanoma of the oral cavity Ebenezer J - Indian J ... 2016;7-11.

8. Uratani AM, Vargas PA, Jorge J. Oral melanoma: review of the literature. 2004;3:428-32.

9. S N, MN V, S B, F F. Oral Malignant Melanoma: A Brief Review. J Clin Exp Pathol. 2016;06.

10. Saigal K, Weed DT, Reis IM, Markoe AM, Wolfson AH, Nguyen-sperry J. Mucosal Melanomas of the Head and Neck: The Role of Postoperative Radiation Therapy. 2012;2012:1-7.

11. Bhatia S, Tykodi SS, Thompson JA. Treatment of metastatic melanoma: an overview. Oncology (Williston Park). 2009;23:488-96.

12. D'souza J, Padhye A. Oral malignant melanoma: A silent killer? J Indian Soc Periodontol. 2011;15:425. 